\section{Author Correction: Slower decay of landfalling hurricanes in a warming world}

https://doi.org/10.1038/s41586-021-03321-9

Correction to: Nature https://doi.org/10.1038/s41586-020-2867-7

Published online 11 November 2020

Check for updates

Lin Li \& Pinaki Chakraborty

In the Article, we studied 71 landfall events. For each event, we analysed the data of hurricane intensities and locations corresponding to the first four synoptic times past landfall (0000 UTC, 0600 UTC, 1200 UTC and 1800 UTC). From the intensities, we computed the decay timescale, and from the locations, we computed the translation speed, coastline-perpendicular translation speed, and latitude and longitude of the centroid. For five landfall events (Jerry in 1989; Georges in 1998; Ivan in 2004; Dolly in 2008; and Richard in 2010), we mistakenly analysed the data for one asynoptic time by considering it as synoptic (see the times marked in boldface in Table 1 of this Amendment). As a result, the reported values of the decay timescale, translation speed, coastline-perpendicular translation speed, and latitude and longitude of the centroid for these five landfall events are incorrect. This affects Fig. 1b, c, e, f, Fig. 3, Extended Data Fig. 1b, d, f, Extended Data Fig. 2a, b, d, e, Extended Data Fig. 3, Extended Data Table 2, Extended Data Table 3b, c, Supplementary Data and the Source Data for Fig. 1 of the original Article, and these figures and tables have all been corrected
Table 1 | Synoptic and asynoptic times used in the original and corrected analyses

\begin{tabular}{|c|c|c|}
\hline Landfall event & $\begin{array}{l}\text { Original analysis (time in } \\
\text { UTC) }\end{array}$ & $\begin{array}{l}\text { Corrected analysis (time in } \\
\text { UTC) }\end{array}$ \\
\hline Jerry (1989) & $\begin{array}{l}16 \text { Oct: } 0000, \mathbf{0 0 3 0}, 0600, \\
1200\end{array}$ & $\begin{array}{l}16 \text { Oct: } 0000,0600,1200, \\
1800\end{array}$ \\
\hline Georges (1998) & $\begin{array}{l}\text { 22/23 Sep: } 1200,1230, \\
1800,0000\end{array}$ & $\begin{array}{l}\text { 22/23 Sep: } 1200,1800,0000 \\
0600\end{array}$ \\
\hline Ivan (2004) & $\begin{array}{l}\text { 16/17 Sep: 0650, 1200, } \\
\text { 1800, 0000 }\end{array}$ & $\begin{array}{l}\text { 16/17 Sep: 1200, 1800, 0000, } \\
0600\end{array}$ \\
\hline Dolly (2008) & $\begin{array}{l}\text { 23/24 July: 1800, 1820, } \\
\text { 0000, } 0600\end{array}$ & $\begin{array}{l}23 / 24 \text { July: } 1800,0000,0600, \\
1200\end{array}$ \\
\hline Richard (2010) & $\begin{array}{l}25 \text { Oct: } 0000, \mathbf{0 0 3 0}, 0600, \\
1200\end{array}$ & $\begin{array}{l}25 \text { Oct: } 0000,0600,1200, \\
1800\end{array}$ \\
\hline
\end{tabular}

online. Correspondingly, many of the numerical values reported in the original Article have minor changes; for example, in the legend to Fig.1, the correlation, $r$, between the decay timescale and SST has changed from 0.73 to 0.72 ; in the footnote to Extended Data Table 2, the text 'two-sided $95 \% \mathrm{Cl}$ of the corresponding slope' has changed to 'two-sided 95\% CI (93\% CI for the two cases marked with an asterisk) of the corresponding slope'; and many numerical values in the 'Hurricane tracks and decay' section of the Methods have changed slightly. Figures 1-7 of this Amendment show the incorrect, as-published versions next to the corrected versions, for transparency. The Supplementary Information of this Amendment contains the incorrect, as-published versions of the Supplementary Data and the Source Data for Fig. 1 of the original Article, for transparency. In the corrected analysis, we discarded the data corresponding to the asynoptic time and instead analysed the data for the first four synoptic times past landfall (see the rightmost column in Table 1 of this Amendment). The resulting changes in the values of the decay timescale and other parameters do not affect our discussions and conclusions. We apologize for the error and thank Kelvin Chan for bringing this error to our attention. The original Article has been corrected online. 
Original Fig. 1b,c,e,f

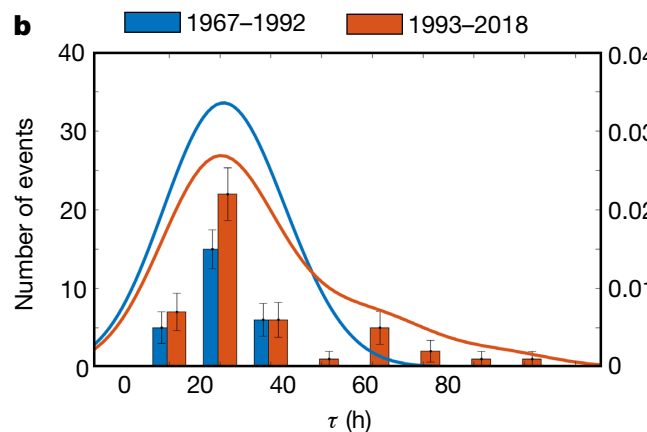

c
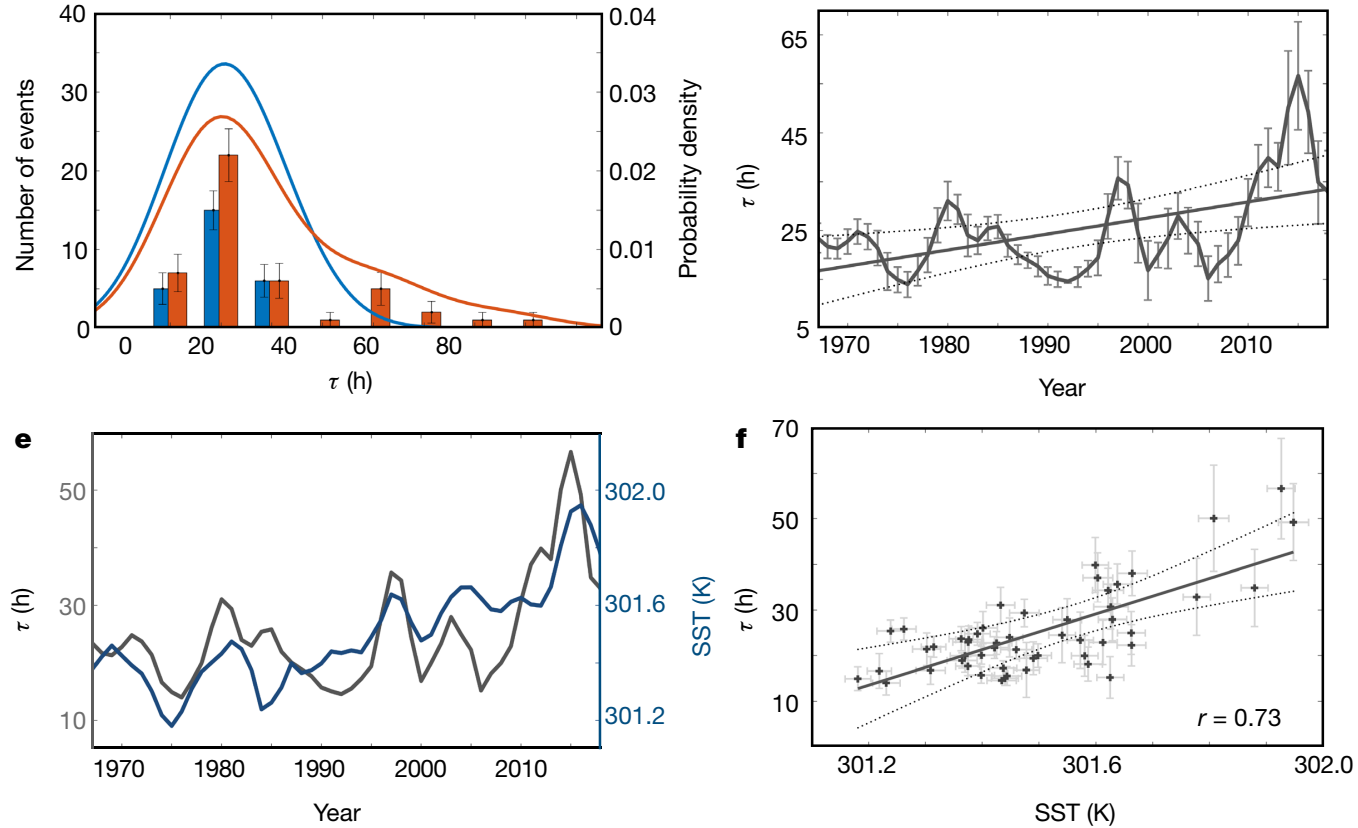

\section{Corrected Fig. 1b,c,e,f}

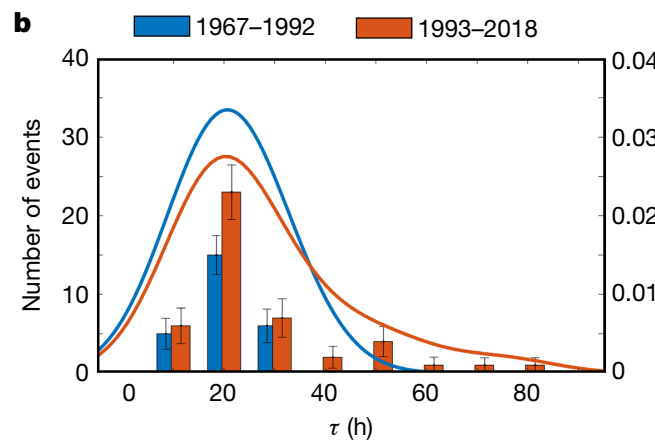

c
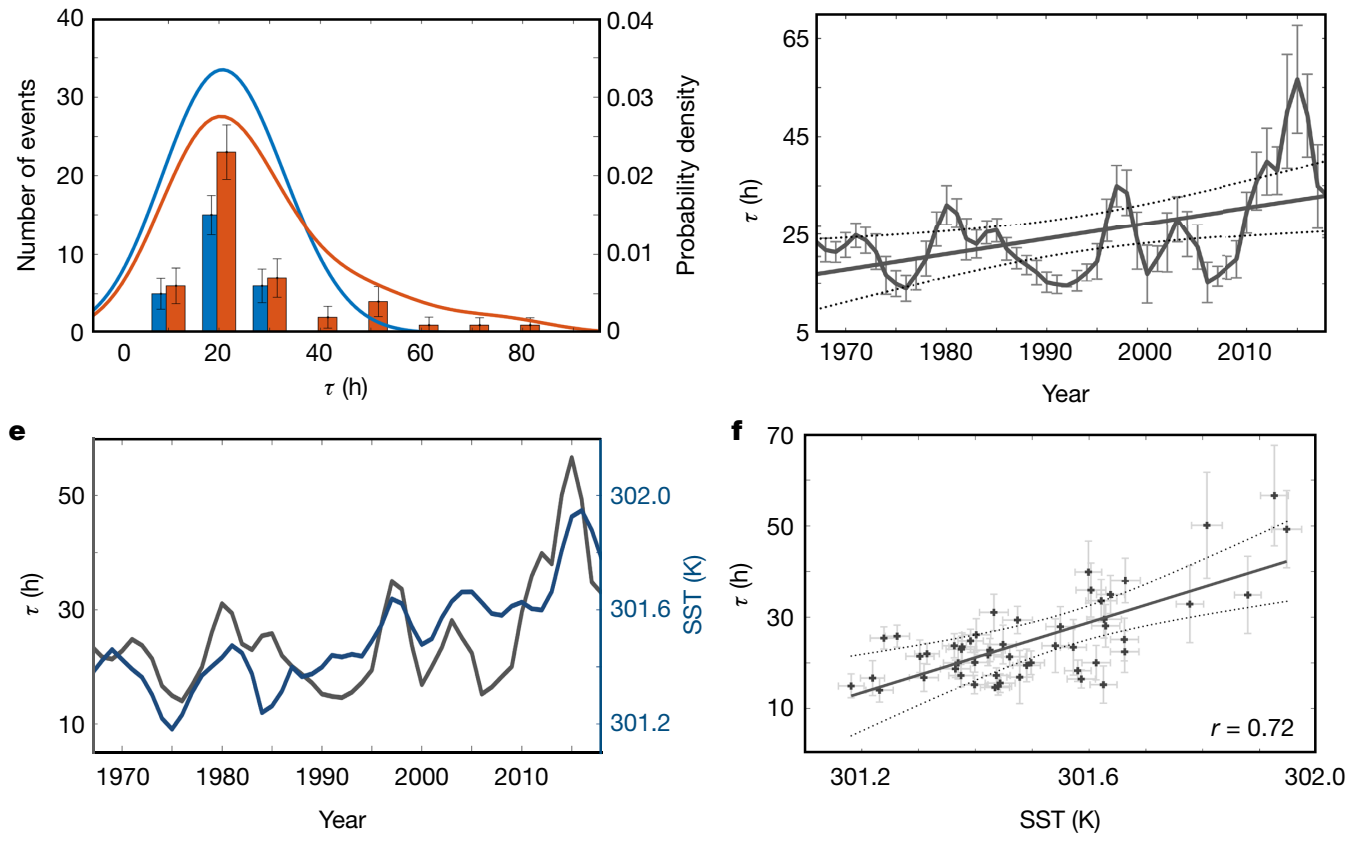

Fig. 1 | This figure shows the incorrect, as-published version and the corrected version of Fig. $1 b, c, e$ and $f$ of the original Article. (Note that the error does not affect the span of $\tau$ in Fig. $1 \mathrm{~b}$; the difference in the spans of the

original and corrected versions shown here is because the abscissa in the original version was incorrect owing to a separate error during production, now also corrected here.) 
Original Fig. 3
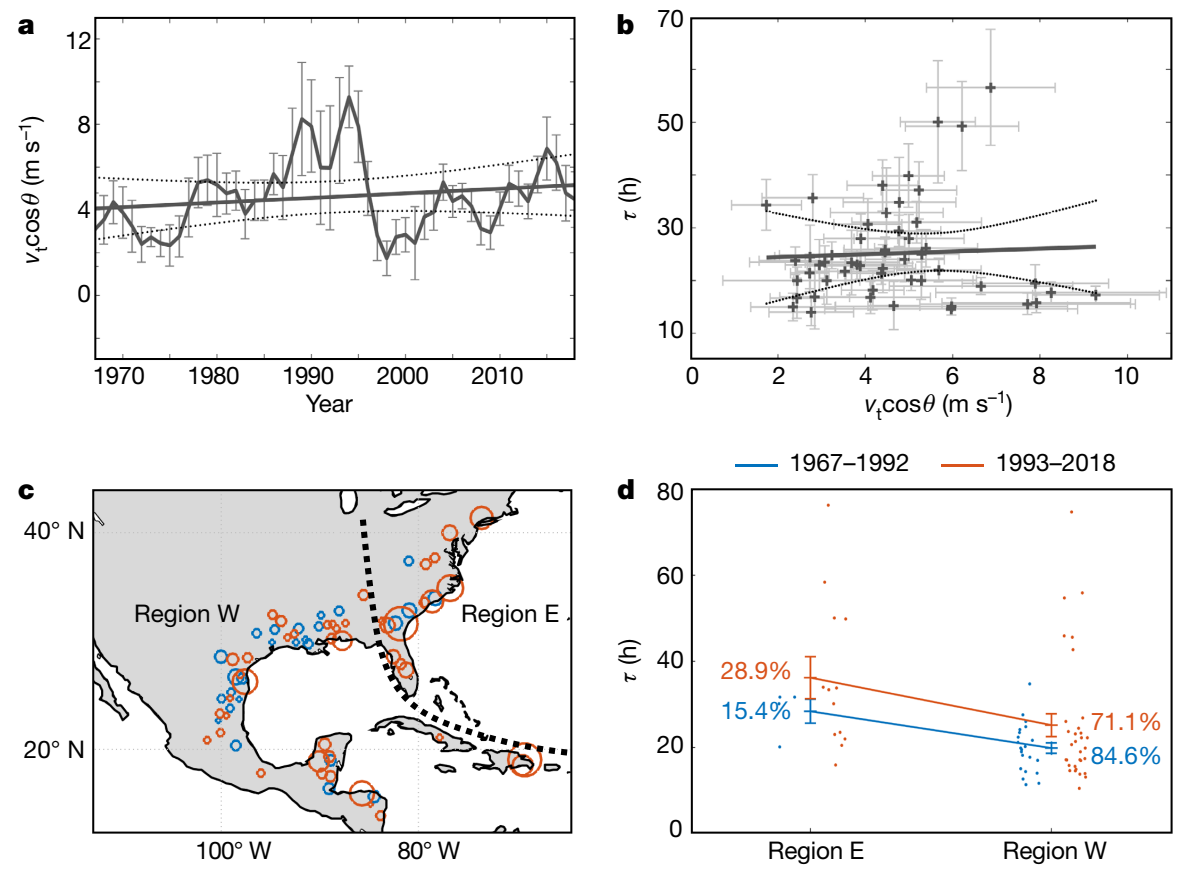

Corrected Fig. 3
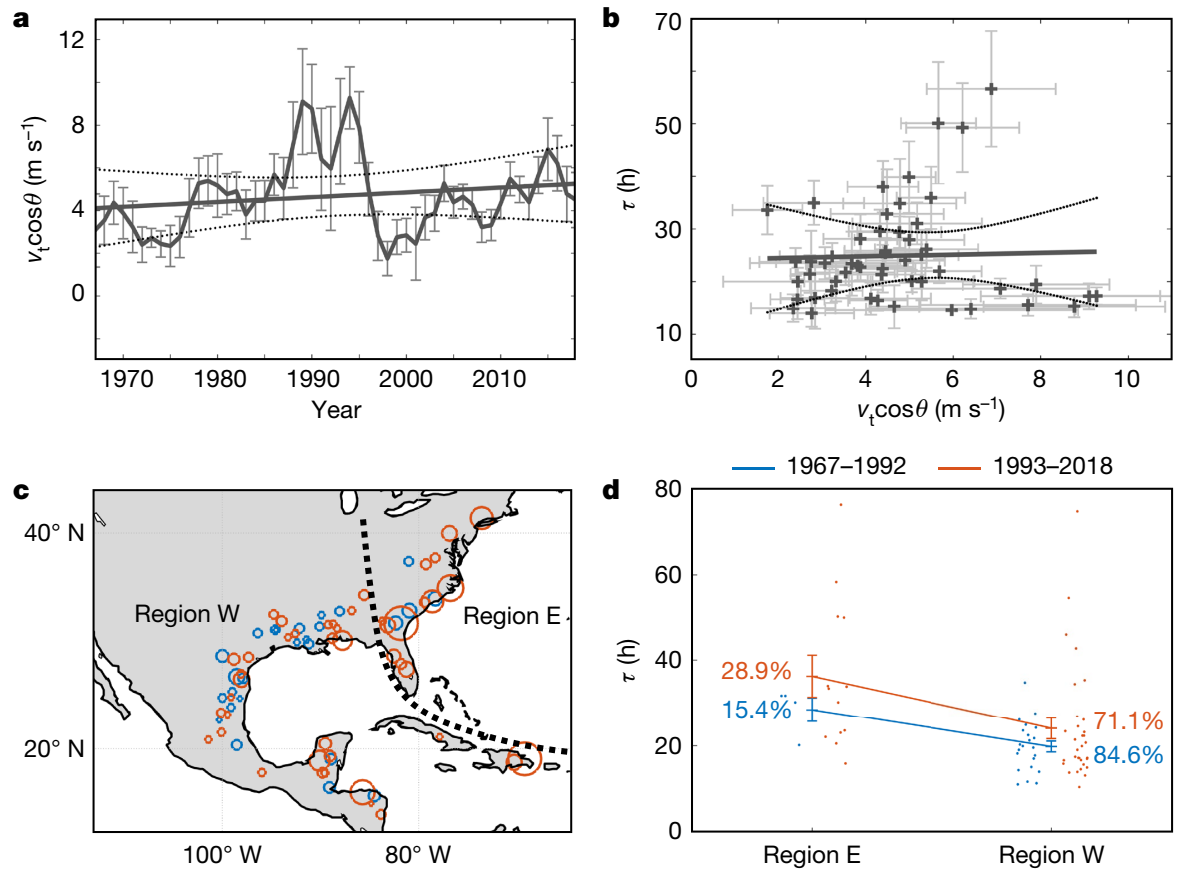

Fig. 2 |This figure shows the incorrect, as-published version and the corrected version of Fig. 3 of the original Article. 
Original Extended Data Fig. 1b,d,f
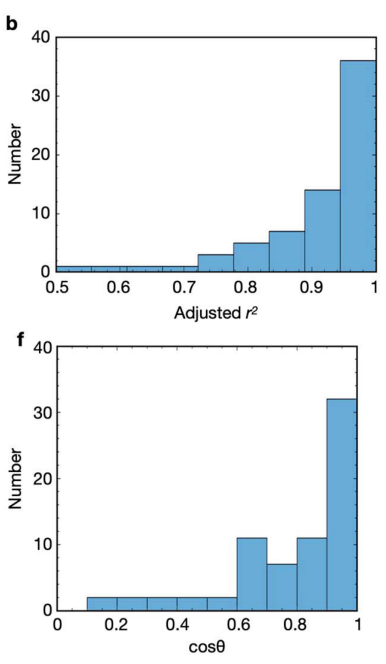

d
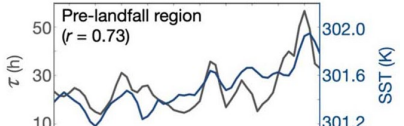

Main Development Regian 301.2

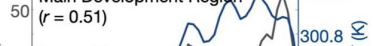

点 ${ }^{30}$

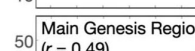

$50(r=0.49)$

$\sum_{\omega} 30$.

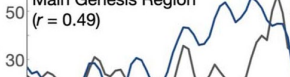

V V 300.4 点

19701980199020002010

Year
Corrected Extended Data Fig. 1b,d,f

b
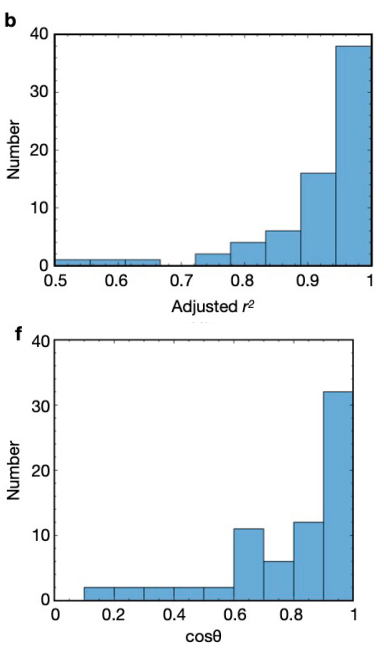
$50 \begin{aligned} & \text { Pre-landfall region } \\ & (r=0.72)\end{aligned} 302.0$

年301.2 ${ }_{50}$ Main Development Region ${ }^{301.2}$ E $307=0.48)$

Main Genesis Region 301.2 $50 \substack{(r=0.46) \\ \text { Main Genesis Region }}_{300.8}^{301.2}$ E ${ }^{30} 300.8 \frac{8}{5}$ 19701980199020002010

Fig. 3 | This figure shows the incorrect, as-published version and the corrected version of Extended Data Fig. $1 b$, $d$ and $f$ of the original Article. 
Original Extended Data Fig. 2a,b,d,e

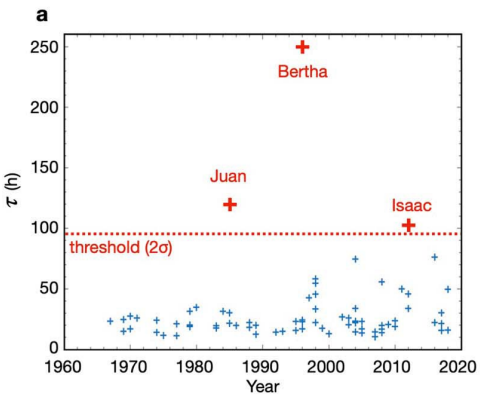

b

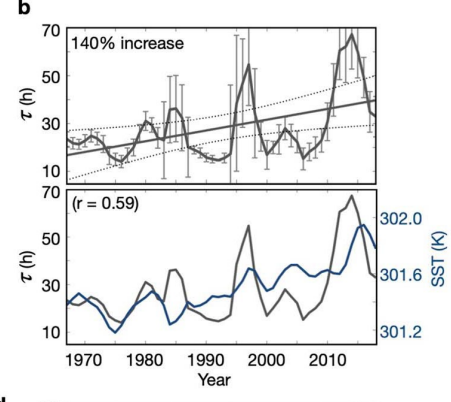

d

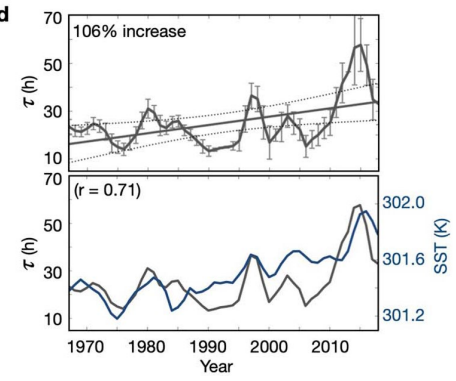

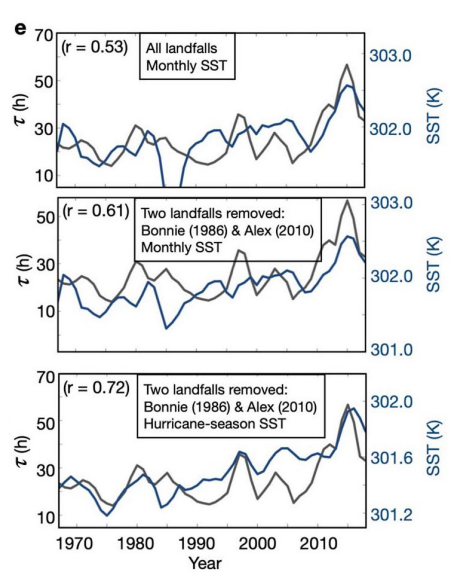

Corrected Extended Data Fig. 2a,b,d,e

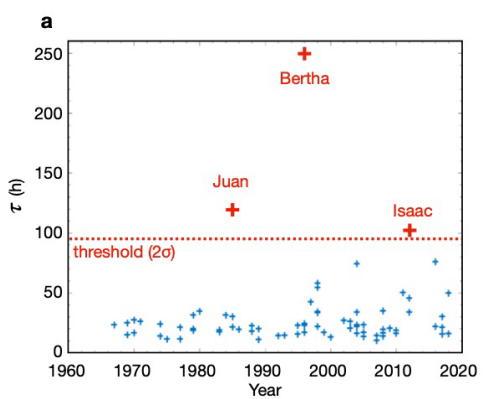

b

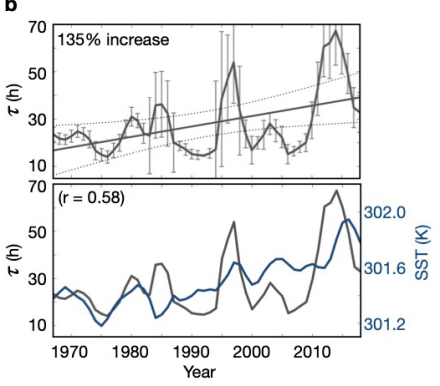

d

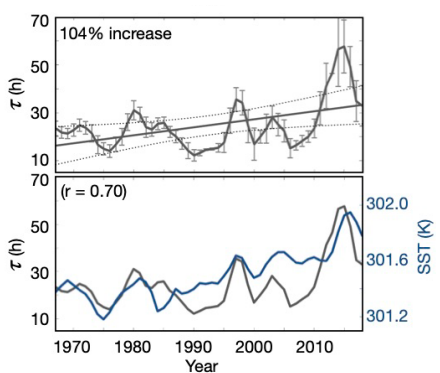

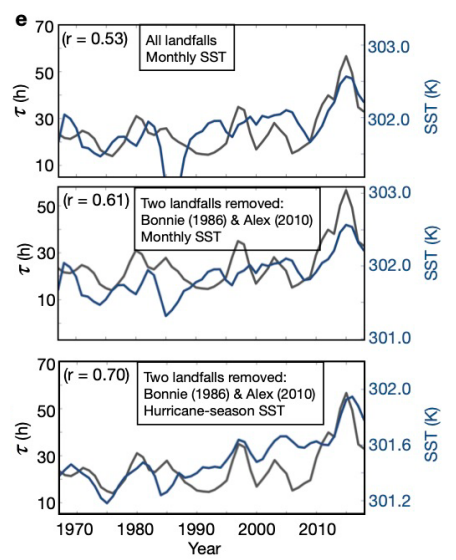

$$
\text { : }
$$

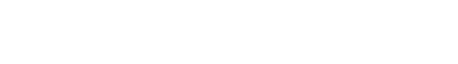


Original Extended Data Fig. 3

a

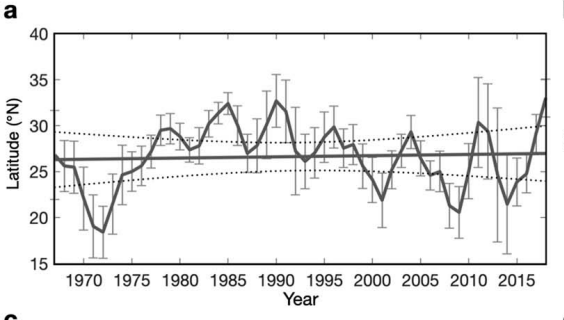

C

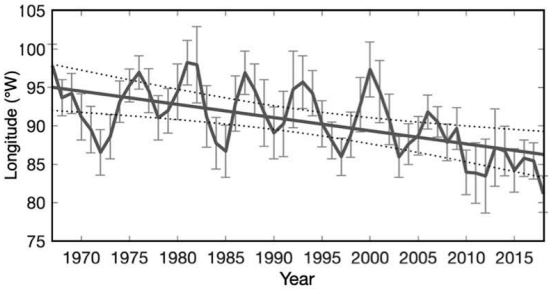

。

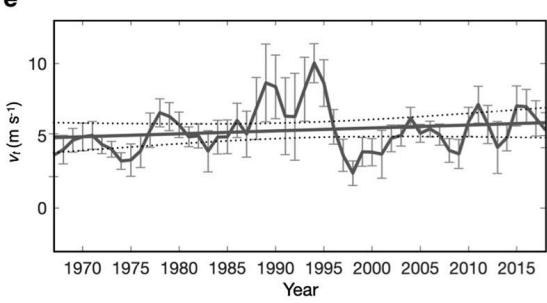

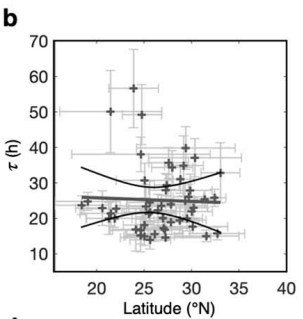

d
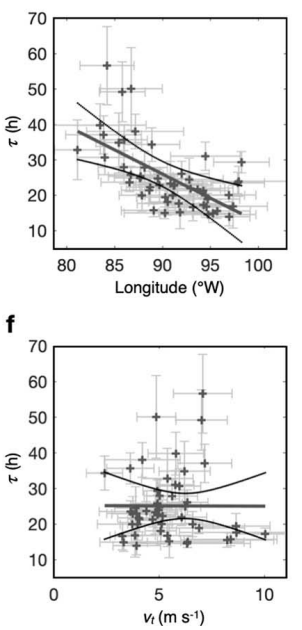

Corrected Extended Data Fig. 3
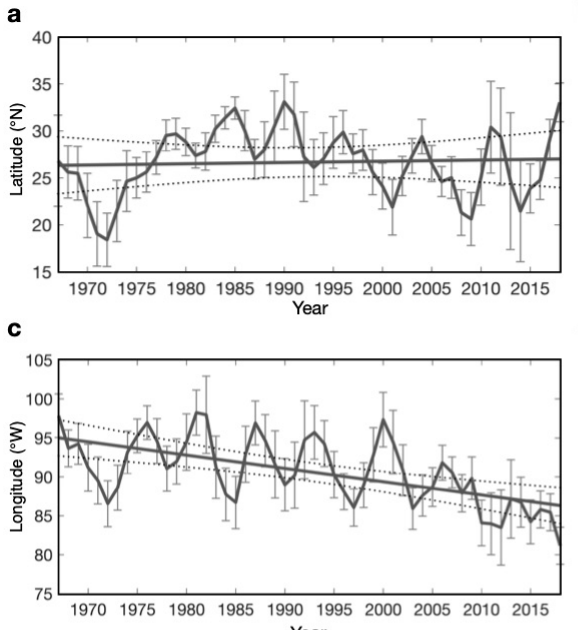

Year

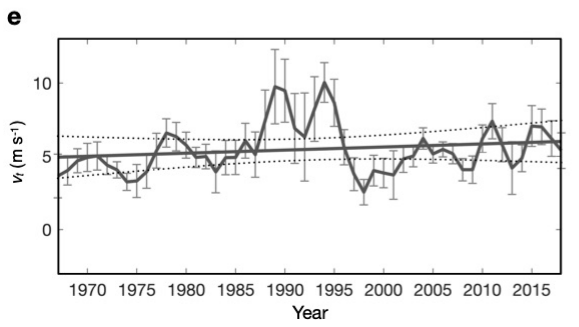

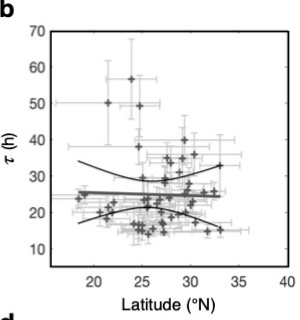

d
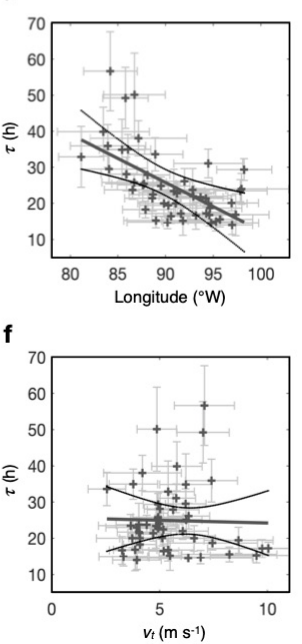

Fig. 5 | This figure shows the incorrect, as-published version and the corrected version of Extended Data Fig. 3 of the original Article. 
Corrections \& amendments

Original Extended Data Table 2

\begin{tabular}{|c|c|c|c|c|c|c|}
\hline & Slope & $\mathrm{Cl}$ & & d.f. & \multicolumn{2}{|c|}{$P$ value (uncorrected) } \\
\hline$\tau$-year & $0.33 \mathrm{~h} / \mathrm{yr}$ & {$[0.04,0.62]$} & & 15 & \multicolumn{2}{|c|}{$<10^{-4}$} \\
\hline SST - year & $0.010 \mathrm{~K} / \mathrm{yr}$ & {$[0.006,0.013]$} & & 15 & \multicolumn{2}{|c|}{$<10^{-13}$} \\
\hline latitude - year & $0.01^{\circ} \mathrm{N} / \mathrm{yr}$ & {$[-0.11,0.14]$} & & 15 & \multicolumn{2}{|c|}{0.68} \\
\hline longitude - year & $-0.17^{\circ} \mathrm{W} / \mathrm{yr}$ & {$[-0.27,-0.08]$} & & 24 & \multicolumn{2}{|c|}{$<10^{-5}$} \\
\hline vt - year & $0.020 \mathrm{~m} \mathrm{~s}^{-1} / \mathrm{yr}$ & {$[-0.023,0.063]$} & & 24 & \multicolumn{2}{|c|}{0.17} \\
\hline vt $\cos \theta-$ year & $0.022 \mathrm{~m} \mathrm{~s}^{-1} / \mathrm{yr}$ & {$[-0.039,0.082]$} & & 15 & \multicolumn{2}{|c|}{0.17} \\
\hline $\begin{array}{l}\tau \text { - year (with outliers); } \\
\text { see Extended Data Fig. 2b }\end{array}$ & $0.45 \mathrm{~h} / \mathrm{yr}$ & {$[0.02,0.88]$} & & 15 & \multicolumn{2}{|c|}{$<10^{-3}$} \\
\hline $\begin{array}{l}\tau \text {-year (without ET); } \\
\text { see Extended Data Fig. 2d }\end{array}$ & $0.34 \mathrm{~h} / \mathrm{yr}$ & {$[0.03,0.67]$} & & 15 & \multicolumn{2}{|c|}{$<10^{-3}$} \\
\hline & Slope & $\mathrm{Cl}$ & d.f. & & $P$-value (uncorrected) & $\mathrm{r}$ \\
\hline$\tau$-SST & $39 \mathrm{~h} / \mathrm{K}$ & {$[19,59]$} & 15 & & $<10^{-9}$ & 0.73 \\
\hline$\tau$-latitude & $-0.1 \mathrm{~h} /{ }^{\circ} \mathrm{N}$ & {$[-1.6,1.4]$} & 15 & & 0.80 & -0.04 \\
\hline$\tau$ - longitude & $-1.4 \mathrm{~h} /{ }^{\circ} \mathrm{W}$ & {$[-2.3,-0.4]$} & 15 & & $<10^{-6}$ & -0.62 \\
\hline$\tau$-vt & $-0.02 \mathrm{~h} /\left(\mathrm{m} \mathrm{s}^{-1}\right)$ & {$[-3.32,3.28]$} & 15 & & 0.98 & -0.003 \\
\hline$\tau-\mathrm{vt} \cos \theta$ & $0.3 \mathrm{~h} /\left(\mathrm{m} \mathrm{s}^{-1}\right)$ & {$[-2.8,3.3]$} & 15 & & 0.74 & 0.05 \\
\hline longitude - SST & $-14^{\circ} \mathrm{W} / \mathrm{K}$ & {$[-25,-4]$} & 15 & & $<10^{-5}$ & -0.59 \\
\hline$\tau$ - SST (detrended) & $51 \mathrm{~h} / \mathrm{K}$ & {$[15,87]$} & 15 & & $<10^{-5}$ & 0.62 \\
\hline$\tau$ - latitude (detrended) & $-0.2 \mathrm{~h} /{ }^{\circ} \mathrm{N}$ & {$[-1.5,1.1]$} & 15 & & 0.58 & -0.08 \\
\hline$\tau$-longitude (detrended) & $-1.0 \mathrm{~h} /{ }^{\circ} \mathrm{W}$ & {$[-2 \cdot 2,0.1]$} & 15 & & $<10^{-3}$ & -0.05 \\
\hline$\tau$ - vt (detrended) & $-0.7 \mathrm{~h} /\left(\mathrm{m} \mathrm{s}^{-1}\right)$ & {$[-3.5,2.2]$} & 15 & & 0.37 & -0.13 \\
\hline$\tau-\mathrm{vt} \cos \theta$ (detrended) & $-0.3 \mathrm{~h} /\left(\mathrm{m} \mathrm{s}^{-1}\right)$ & {$[-3.0,2.3]$} & 15 & & 0.65 & -0.07 \\
\hline longitude - SST (detrended) & $-7^{\circ} \mathrm{W} / \mathrm{K}$ & {$[-26,12]$} & 15 & & 0.17 & -0.19 \\
\hline $\begin{array}{l}\tau \text { - SST (from MDR); } \\
\text { see Extended Data Fig. 1d }\end{array}$ & $18 \mathrm{~h} / \mathrm{K}$ & {$[1,36]$} & 15 & & $<10^{-3}$ & 0.51 \\
\hline $\begin{array}{l}\tau \text { - SST (from MGR); } \\
\text { see Extended Data Fig 1d }\end{array}$ & $17 \mathrm{~h} / \mathrm{K}$ & {$[0.1,33]$} & 15 & & $<10^{-3}$ & 0.49 \\
\hline $\begin{array}{l}\tau \text {-SST (with outliers); } \\
\text { see Extended Data Fig. 2b }\end{array}$ & $45 \mathrm{~h} / \mathrm{K}$ & {$[11,80]$} & 15 & & $<10^{-5}$ & 0.59 \\
\hline $\begin{array}{l}\tau \text {-(monthly) SST; } \\
\text { see Extended Data Fig. 2c }\end{array}$ & $15 \mathrm{~h} / \mathrm{K}$ & {$[2,28]$} & 15 & & $<10^{-4}$ & 0.53 \\
\hline $\begin{array}{l}\tau \text { - (monthly) SST (- } 2 \text { landfalls); } \\
\text { see Extended Data Fig. } 2 c\end{array}$ & $21 \mathrm{~h} / \mathrm{K}$ & {$[6,36]$} & 15 & & $<10^{-5}$ & 0.61 \\
\hline $\begin{array}{l}\tau \text { - SST (- } 2 \text { landfalls); } \\
\text { see Extended Data Fig. 2c }\end{array}$ & $39 \mathrm{~h} / \mathrm{K}$ & {$[18,59]$} & 15 & & $<10^{-8}$ & 0.72 \\
\hline $\begin{array}{l}\tau \text {-SST (without ET); } \\
\text { see Extended Data Fig. 2d }\end{array}$ & $41 \mathrm{~h} / \mathrm{K}$ & {$[19,64]$} & 15 & & $<10^{-8}$ & 0.71 \\
\hline
\end{tabular}

Corrected Extended Data Table 2

\begin{tabular}{|c|c|c|c|c|c|c|}
\hline & Slope & $\mathrm{Cl}$ & & d.f. & \multicolumn{2}{|c|}{$P$ value (uncorrected) } \\
\hline$\tau$ - year & $0.32 \mathrm{~h} / \mathrm{yr}$ & {$[0.02,0.61]$} & & 15 & \multicolumn{2}{|c|}{$<10^{-3}$} \\
\hline SST - year & $0.010 \mathrm{~K} / \mathrm{yr}$ & {$[0.006,0.013]$} & & 15 & \multicolumn{2}{|c|}{$<10^{-13}$} \\
\hline latitude - year & $0.01{ }^{\circ} \mathrm{N} / \mathrm{yr}$ & {$[-0.11,0.14]$} & & 15 & \multicolumn{2}{|c|}{0.68} \\
\hline longitude - year & $-0.17^{\circ} \mathrm{W} / \mathrm{yr}$ & {$[-0.27,-0.08]$} & & 24 & \multicolumn{2}{|c|}{$<10^{-5}$} \\
\hline vt - year & $0.021 \mathrm{~m} \mathrm{~s}^{-1} / \mathrm{yr}$ & {$[-0.038,0.081]$} & & 15 & \multicolumn{2}{|c|}{0.18} \\
\hline vt $\cos \theta-$ year & $.022 \mathrm{~m} \mathrm{~s}^{-1 / y r}$ & {$[-0.054,0.099]$} & & 11 & \multicolumn{2}{|c|}{0.17} \\
\hline $\begin{array}{l}\tau \text { - year (with outliers); } \\
\text { see Extended Data Fig. 2b }\end{array}$ & $0.44 \mathrm{~h} / \mathrm{yr}$ & {$[0.003,0.88]$} & & 15 & \multicolumn{2}{|c|}{$<10^{-3}$} \\
\hline $\begin{array}{l}\tau \text { - year (without ET); } \\
\text { see Extended Data Fig. 2d }\end{array}$ & $0.33 \mathrm{~h} / \mathrm{yr}$ & {$[0.004,0.66]$} & & 15 & \multicolumn{2}{|c|}{$<10^{-3}$} \\
\hline & Slope & $\mathrm{Cl}$ & d.f. & & D-value (uncorrected) & $r$ \\
\hline$\tau$-SST & $38 \mathrm{~h} / \mathrm{K}$ & {$[18,59]$} & 15 & & $<10^{-8}$ & 0.72 \\
\hline$\tau$ - latitude & $-0.1 \mathrm{~h} /{ }^{\circ} \mathrm{N}$ & {$[-1.6,1.4]$} & 15 & & 0.83 & -0.03 \\
\hline$\tau$ - longitude & $-1.3 \mathrm{~h} / \% \mathrm{~W}$ & {$[-2.3,-0.4]$} & 15 & & $<10.5$ & -0.61 \\
\hline$\tau-\mathrm{vt}$ & $-0.15 \mathrm{~h} /\left(\mathrm{m} \mathrm{s}^{-1}\right)$ & {$[-3.26,2.95]$} & 15 & & 0.85 & -0.027 \\
\hline$\tau$ - vt $\cos \theta$ & $0.2 \mathrm{~h} /\left(\mathrm{m} \mathrm{s}^{-1}\right)$ & {$[-3.4,3.7]$} & 11 & & 0.83 & 0.03 \\
\hline longitude - SST & $-14^{\circ} \mathrm{W} / \mathrm{K}$ & {$[-25,-4]$} & 15 & & $<10^{-5}$ & -0.59 \\
\hline$\tau$-SST (detrended) & $53 \mathrm{~h} / \mathrm{K}$ & {$[16,89]$} & 15 & & $<10^{-6}$ & 0.62 \\
\hline$\tau$ - latitude (detrended) & $-0.2 \mathrm{~h} /{ }^{\circ} \mathrm{N}$ & {$[-1.5,1.1]$} & 15 & & 0.63 & -0.07 \\
\hline$\tau$ - longitude (detrended) & $-1.0 \mathrm{~h} /{ }^{\circ} \mathrm{W}$ & {$[-2.2,0.1]$} & 15 & & $<10^{-2}$ & -0.44 \\
\hline$\tau$-vt (detrended) & $-0.7 \mathrm{~h} /\left(\mathrm{m} \mathrm{s}^{-1}\right)$ & {$[-3.4,2.0]$} & 15 & & 0.30 & -0.15 \\
\hline$\tau-$ vt $\cos \theta$ (detrended) & $-0.4 \mathrm{~h} /\left(\mathrm{m} \mathrm{s}^{-1}\right)$ & {$[-3.5,2.7]$} & 11 & & 0.58 & -0.08 \\
\hline longitude - SST (detrended) & $-7^{\circ} \mathrm{W} / \mathrm{K}$ & {$[-26,12]$} & 15 & & 0.17 & -0.19 \\
\hline $\begin{array}{l}\tau \text { - SST (from MDR); } \\
\text { see Extended Data Fig. 1d }\end{array}$ & $17.5 \mathrm{~h} / \mathrm{K}$ & {$[1.3,33.6]^{\star}$} & 15 & & $<10^{-3}$ & 0.48 \\
\hline $\begin{array}{l}\tau \text { - SST (from MGR); } \\
\text { see Extended Data Fig 1d }\end{array}$ & $16 \mathrm{~h} / \mathrm{K}$ & {$[0.3,31.1]^{*}$} & 15 & & $<10 \cdot 3$ & 0.46 \\
\hline $\begin{array}{l}\tau \text {-SST (with outliers); } \\
\text { see Extended Data Fig. 2b }\end{array}$ & $45 \mathrm{~h} / \mathrm{K}$ & {$[10,80]$} & 15 & & $<10.5$ & 0.58 \\
\hline $\begin{array}{l}\tau \text { - (monthly) SST; } \\
\text { see Extended Data Fig. 2c }\end{array}$ & $15 \mathrm{~h} / \mathrm{K}$ & {$[2,28]$} & 15 & & $<10^{-4}$ & 0.53 \\
\hline $\begin{array}{l}\tau \text { - (monthly) SST (- } 2 \text { landfalls); } \\
\text { see Extended Data Fig. 2c }\end{array}$ & $21 \mathrm{~h} / \mathrm{K}$ & {$[6,36]$} & 15 & & $<10^{-5}$ & 0.61 \\
\hline $\begin{array}{l}\tau \text { - SST (- } 2 \text { landfalls); } \\
\text { see Extended Data Fig. 2c }\end{array}$ & $38 \mathrm{~h} / \mathrm{K}$ & {$[17,59]$} & 15 & & $<10-8$ & 0.71 \\
\hline $\begin{array}{l}\tau \text { - SST (without ET); } \\
\text { see Extended Data Fig. 2d }\end{array}$ & $41 \mathrm{~h} / \mathrm{K}$ & {$[18,64]$} & 15 & & $<10^{-7}$ & 0.70 \\
\hline
\end{tabular}

Fig. 6 | This figure shows the incorrect, as-published version and the corrected version of Extended Data Table 2 of the original Article. 
Original Extended Data Table 3b,c
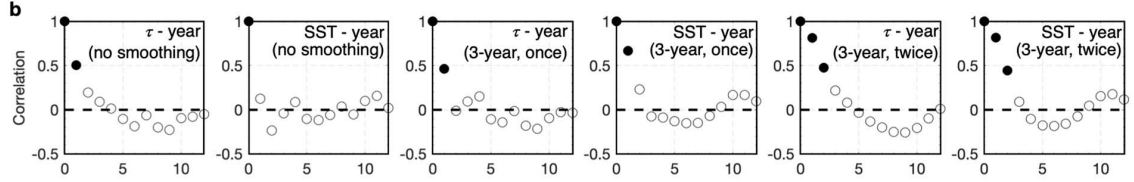

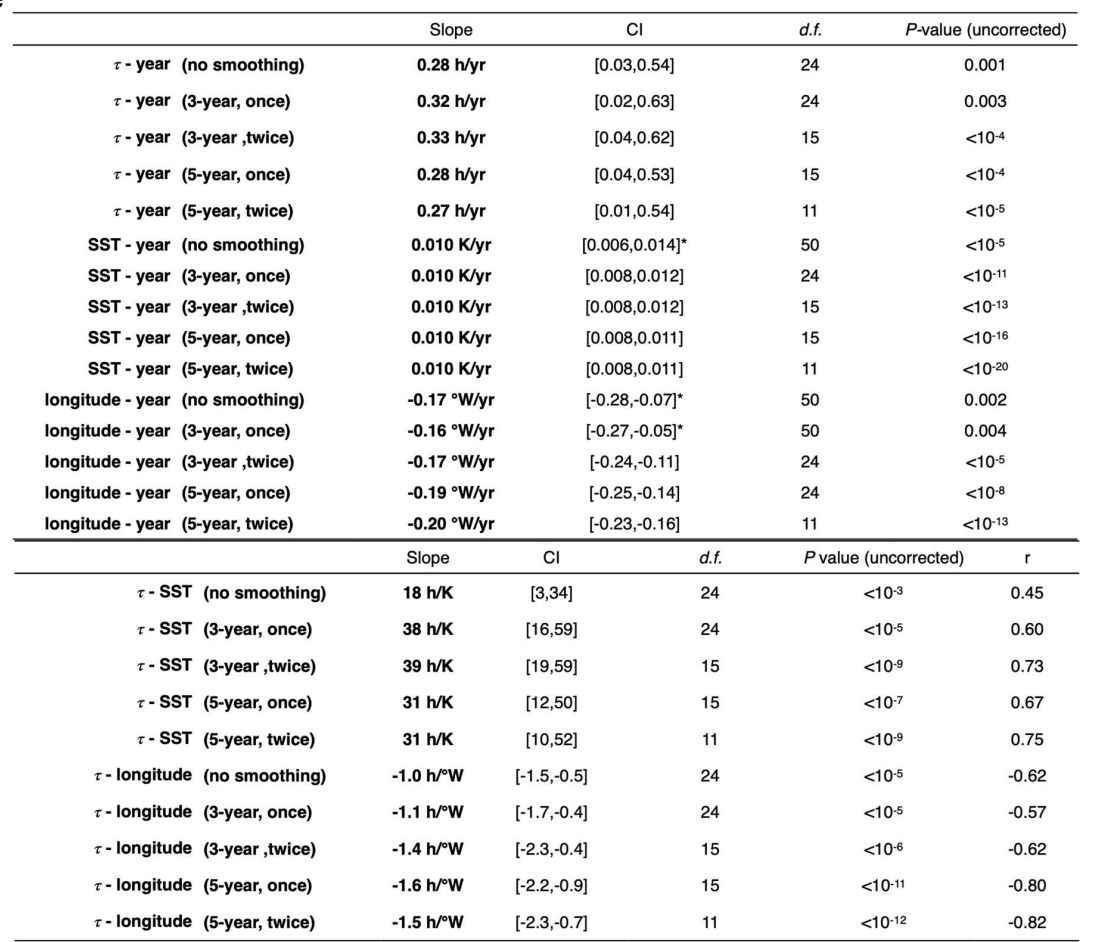

\section{Corrected Extended Data Table 3b,c}
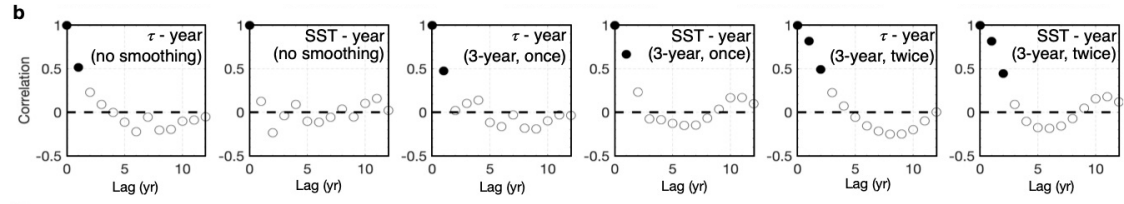

\begin{tabular}{|c|c|c|c|c|c|c|}
\hline & Slope & \multicolumn{2}{|r|}{$\mathrm{Cl}$} & d.f. & \multicolumn{2}{|c|}{$P$-value (uncorrected) } \\
\hline$\tau$ - year (no smoothing) & $0.28 \mathrm{~h} / \mathrm{yr}$ & \multicolumn{2}{|r|}{$[0.02,0.53]$} & 24 & \multicolumn{2}{|r|}{0.002} \\
\hline$\tau$-year (3-year, once) & $0.31 \mathrm{~h} / \mathrm{yr}$ & \multicolumn{2}{|r|}{$[0.004,0.62]$} & 24 & \multicolumn{2}{|r|}{0.004} \\
\hline$\tau$-year (3-year , twice) & $0.32 \mathrm{~h} / \mathrm{yr}$ & \multicolumn{2}{|r|}{$[0.02,0.61]$} & 15 & \multicolumn{2}{|r|}{$<10^{-3}$} \\
\hline$\tau$-year (5-year, once) & $0.27 \mathrm{~h} / \mathrm{yr}$ & \multicolumn{2}{|r|}{$[0.02,0.52]$} & 15 & \multicolumn{2}{|r|}{$<10^{-4}$} \\
\hline$\tau$ - year (5-year, twice) & $0.26 \mathrm{~h} / \mathrm{yr}$ & \multicolumn{2}{|r|}{$[0.02,0.50]$} & 11 & \multicolumn{2}{|r|}{$<10^{-5}$} \\
\hline SST - year (no smoothing) & $0.010 \mathrm{~K} / \mathrm{yr}$ & \multicolumn{2}{|r|}{$[0.006,0.014]^{*}$} & 50 & \multicolumn{2}{|r|}{$<10^{-5}$} \\
\hline SST - year (3-year, once) & $0.010 \mathrm{~K} / \mathrm{yr}$ & \multicolumn{2}{|r|}{$[0.008,0.012]$} & 24 & \multicolumn{2}{|r|}{$<10^{-11}$} \\
\hline SST - year (3-year, twice) & $0.010 \mathrm{~K} / \mathrm{yr}$ & \multicolumn{2}{|r|}{$[0.008,0.012]$} & 15 & \multicolumn{2}{|r|}{$<10^{-13}$} \\
\hline SST - year (5-year, once) & $0.010 \mathrm{~K} / \mathrm{yr}$ & \multicolumn{2}{|r|}{$[0.008,0.011]$} & 15 & \multicolumn{2}{|r|}{$<10^{-16}$} \\
\hline SST - year (5-year, twice) & $0.010 \mathrm{~K} / \mathrm{yr}$ & \multicolumn{2}{|r|}{$[0.008,0.011]$} & 11 & \multicolumn{2}{|r|}{$<10^{-20}$} \\
\hline longitude - year (no smoothing) & $-0.17^{\circ} \mathrm{W} / \mathrm{yr}$ & \multicolumn{2}{|r|}{$[-0.28,-0.07]^{*}$} & 50 & \multicolumn{2}{|r|}{0.002} \\
\hline longitude - year (3-year, once) & $-0.16^{\circ} \mathrm{W} / \mathrm{yr}$ & \multicolumn{2}{|r|}{$[-0.27,-0.05]^{\star}$} & 50 & \multicolumn{2}{|r|}{0.005} \\
\hline longitude - year (3-year, twice) & $-0.17^{\circ} \mathrm{W} / \mathrm{yr}$ & \multicolumn{2}{|r|}{$[-0.27,-0.08]$} & 24 & \multicolumn{2}{|r|}{$<10^{-5}$} \\
\hline longitude - year (5-year, once) & $-0.19^{\circ} \mathrm{W} / \mathrm{yr}$ & \multicolumn{2}{|r|}{$[-0.27,-0.11]$} & 24 & \multicolumn{2}{|r|}{$<10^{-8}$} \\
\hline \multirow[t]{2}{*}{ longitude - year (5-year, twice) } & $-0.20^{\circ} \mathrm{W} / \mathrm{yr}$ & \multicolumn{2}{|r|}{$[-0.28,-0.11]$} & 11 & & $<10^{-13}$ \\
\hline & Slope & $\mathrm{Cl}$ & d.f & $P$ valu & ue (uncorrected) & $r$ \\
\hline$\tau$-SST (no smoothing) & $18 \mathrm{~h} / \mathrm{K}$ & {$[3,33]$} & 24 & & $<10 \cdot 3$ & 0.45 \\
\hline$\tau$-SST (3-year, once) & $37 \mathrm{~h} / \mathrm{K}$ & {$[16,59]$} & 24 & & $<10^{-5}$ & 0.59 \\
\hline$\tau$-SST (3-year, twice) & $38 \mathrm{~h} / \mathrm{K}$ & {$[18,59]$} & 15 & & $<10^{-8}$ & 0.72 \\
\hline$\tau$-SST (5-year, once) & $31 \mathrm{~h} / \mathrm{K}$ & {$[11,50]$} & 15 & & $<10^{-6}$ & 0.66 \\
\hline$\tau$-SST (5-year, twice) & $30 \mathrm{~h} / \mathrm{K}$ & {$[11,49]$} & 11 & & $<10^{-9}$ & 0.73 \\
\hline$\tau$ - longitude (no smoothing) & $-1.0 h^{\circ} W$ & {$[-1.5,-0.5]$} & 24 & & $<10^{-5}$ & -0.61 \\
\hline$\tau$-longitude (3-year, once) & $-1.1 \mathrm{~h} /{ }^{\circ} \mathrm{W}$ & {$[-1.7,-0.4]$} & 24 & & $<10^{-4}$ & -0.57 \\
\hline$\tau$-longitude (3-year, twice) & $-1.3 h^{\circ} W$ & {$[-2.3,-0.4]$} & 15 & & $<10^{-5}$ & -0.61 \\
\hline$\tau$-longitude (5-year, once) & $-1.5 h^{\circ} \mathrm{W}$ & {$[-2.2,-0.9]$} & 15 & & $<10^{-11}$ & -0.78 \\
\hline$\tau$-longitude (5-year, twice) & $-1.5 h^{\circ}{ }^{\circ} \mathrm{W}$ & {$[-2.2,-0.7]$} & 11 & & $<10^{-11}$ & -0.80 \\
\hline
\end{tabular}

Fig. 7| This figure shows the incorrect, as-published version and the corrected version of Extended Data Table $3 b$ and $c$ of the original Article. 